\title{
Sonic Boom Analysis under Conditions of Atmospheric Uncertainty Using Polynomial Chaos
}

\author{
By Shinkyu Jeong, ${ }^{1)}$ Daichi Ono, ${ }^{2)}$ Koji ShImoyama ${ }^{2)}$ and Atsushi Hashimoto ${ }^{3)}$ \\ ${ }^{1)}$ Department of Mechanical Engineering, Kyunghee University, Yongin, Korea \\ ${ }^{2)}$ Institute of Fluid Science, Tohoku University, Sendai, Japan \\ 3) Japan Aerospace Exploration Agency, Chofu, Japan
}

(Received May 17th, 2012)

\begin{abstract}
This study establishes an efficient approach for sonic boom analysis under conditions of atmospheric uncertainty, such as temperature, density and wind velocity. This approach combines a non-intrusive polynomial chaos (NIPC) method, which approximates the statistical behavior of output function under conditions of uncertainty, with the sonic boom analysis method using an augmented Burgers' equation, which is able to account for the rise time of the sonic boom signature. The present simulations demonstrate the superior capability of NIPC, which can estimate the statistical signature of sonic boom under conditions of atmospheric uncertainty, while ensuring the same accuracy and much less computational time compared to the Monte Carlo (MC) method. The present simulation results indicate that temperature uncertainty has an impact on the local rise in sonic boom pressure, and atmospheric humidity uncertainty has an impact on the entire shape of the sonic boom signature, while wind uncertainty has almost no impact.
\end{abstract}

Key Words: Sonic Boom, Augmented Burgers’ Equation, Atmospheric Uncertainty, Polynomial Chaos

\section{Nomenclature}

$\alpha^{*}$ : output function in NIPC

$\vec{x}_{0}$ : independent deterministic variable in space

$t_{0}$ : independent deterministic variable in time

$\vec{\xi}$ : random variables with uncertainty

$\alpha_{j}: j$-th coefficient of polynomial chaos

$\Psi_{j}: j$-th orthogonal polynomial

$p$ : sound pressure $(\mathrm{Pa})$

$P$ : order of polynomial

$N P$ : number of sample points

$w(\vec{\xi})$ : weight function

$x$ : ray path distance $(\mathrm{m})$

A: ray tube area $\left(\mathrm{m}^{2}\right)$

$c_{0}$ : small-signal sound velocity $(\mathrm{m} / \mathrm{s})$

$\rho_{0}:$ ambient density $\left(\mathrm{kg} / \mathrm{m}^{3}\right)$

$\beta$ : coefficient of nonlinearity

$(\Delta c)_{i}$ : small-signal sound speed increment at $i$-th layer

$\tau_{i}$ : relaxation time at $i$-th layer

$t^{\prime}$ : retarded time

$\delta$ : diffusivity of sound

\section{Introduction}

In the design of low-boom supersonic aircraft, the waveform parameter method ${ }^{1)}$ has been widely used to estimate the strength of sonic boom. However, this method is unable to take into consideration the sonic boom rise time, which has a psychological impact on humans. To evaluate the effect of sonic boom precisely, the rise time should also be taken into account. Recently, a method for solving an

(C) 2013 The Japan Society for Aeronautical and Space Sciences augmented Burgers' equation ${ }^{2,3)}$ is proposed to evaluate sonic boom propagation. This method accounts for thermal viscosity and relaxation effects in wave propagation, making it possible to estimate the sonic boom rise time.

Another issue in sonic boom estimation is the sonic boom characteristics under conditions of atmospheric uncertainty, such as fluctuations of temperature, wind velocity and wind direction. It is known that the intensity and rise time of sonic boom are affected by atmospheric uncertainties. Thus, if a low-boom aircraft is designed under ideal flight conditions without any consideration of these uncertainties, its performance in actual flights may be lower than expected. Therefore, it is necessary to design a low-boom aircraft taking atmospheric uncertainties into consideration.

The method used to determine how likely certain output will be when some aspects of the system are not precisely known is called "uncertainty quantification (UQ)." A general approach for UQ is to sample the output function under many conditions, and then estimate the statistics (mean value and standard deviation) of output function. Recently, many methods for UQ have been introduced, such as Monte Carlo, ${ }^{4)}$ polynomial chaos, ${ }^{5,6)}$ perturbation method, ${ }^{7,8)}$ moment method, ${ }^{9)}$ and the reduced quadrature technique, ${ }^{10)}$ etc. However, the most important requirement for UQ is computational time for practical use because statistical analysis is required.

In this study, the non-intrusive polynomial chaos (NIPC) method is adopted to estimate the statistics of sonic boom propagation under conditions of atmospheric uncertainty. An augmented Burgers' equation is also used to estimate the sonic boom rise time. First, validation of the NIPC method is performed by comparing the statistics obtained 
from the NIPC and Monte Carlo (MC) methods ${ }^{4}$ using a 1D Burgers' equation problem. Then, sonic boom characteristics under conditions of atmospheric temperature, wind and humidity uncertainty are compared and discussed.

\section{NIPC}

\subsection{Method}

NIPC is a probabilistic method, which expresses the behavior of an output function against input uncertainties as a superposition of orthogonal polynomials as follows

$$
\alpha^{*}\left(\vec{x}_{0}, t_{0}, \vec{\xi}\right) \approx \sum_{j=0}^{P} \alpha_{j}\left(\vec{x}_{0}, t_{0}\right) \Psi_{j}(\vec{\xi}) .
$$

This method considers an output function $\alpha^{*}$ as a function of independent deterministic variables $\vec{x}_{0}$ and $t_{0}$, and a random variable $\vec{\xi}=\left(\xi_{1}, \cdots, \xi_{n}\right)$ representing input uncertainties. $\alpha_{j}$ and $\Psi_{j}$ are $j$-th mode of the coefficient and orthogonal polynomial of NIPC, respectively, and $P$ equals the order of polynomial.

It decomposes $\alpha^{*}$ into deterministic (mean) and stochastic (standard deviation) components to estimate the behavior of the output function under conditions of uncertainty. In sonic boom analysis, $\alpha^{*}$ and $\vec{\xi}$ represent the pressure of sonic boom and atmospheric uncertainties, respectively. The type of polynomials used in NIPC is uniquely determined according to probability distribution of input uncertainties $(\vec{\xi})$. Table 1 shows the types of probability distribution of input uncertainties and the corresponding polynomials.
Table 1. Probability distribution of input uncertainties and corresponding polynomials.

\begin{tabular}{llll}
\hline & Distribution $(\xi)$ & Polynomial $(\Psi)$ & \multicolumn{1}{c}{ Support } \\
\hline Continuous & Gaussian & Hermite & $(-\infty, \infty)$ \\
& Gamma & Laguerre & {$[0, \infty)$} \\
& Beta & Jacobi & {$[a, b]$} \\
& Uniform & Legendre & {$[a, b]$} \\
\hline \multirow{2}{*}{ Discrete } & Poisson & Charlier & $(0,1,2, \cdots)$ \\
& Binomial & Krawtchouk & $(0,1,2, \cdots, N)$ \\
& Negative binomial & Meixner & $(0,1,2, \cdots)$ \\
& Hypergeometric & Hahn & $(0,1,2, \cdots, N)$ \\
\hline
\end{tabular}

In this study, distribution is assumed as the Gaussian and the Hermite polynomial is used. The first three polynomials of the Hermite are as follows

$$
\begin{aligned}
& \Psi_{0}(\xi)=1 \\
& \Psi_{1}(\xi)=\xi \\
& \Psi_{2}(\xi)=\xi^{2}-1
\end{aligned}
$$

and the Hermite polynomial also satisfies the following recurrence relationship

$$
\Psi_{n+1}(\xi)=\xi \cdot \Psi_{n}(\xi)-n \cdot \Psi_{n-1}(\xi) .
$$

With $\alpha^{*}\left(\vec{x}_{0}, t_{0}, \vec{\xi}_{j}\right)(j=1, \cdots, N P, N P=$ number of sample points, $N P \geq P+1)$ obtained on samples, the coefficient of NIPC $\alpha_{j}\left(\vec{x}_{0}, t_{0}\right)(j=0,1, \cdots, P)$ can be determined by solving the following system

$$
\left(\begin{array}{ccc}
\Psi_{0}\left(\vec{\xi}_{1}\right) & \cdots & \Psi_{P}\left(\vec{\xi}_{1}\right) \\
\vdots & \ddots & \vdots \\
\Psi_{0}\left(\vec{\xi}_{N P}\right) & \cdots & \Psi_{P}\left(\vec{\xi}_{N P}\right)
\end{array}\right)\left(\begin{array}{c}
\alpha_{0}\left(\vec{x}_{0}, t_{0}\right) \\
\vdots \\
\alpha_{P}\left(\vec{x}_{0}, t_{0}\right)
\end{array}\right)=\left(\begin{array}{c}
\alpha^{*}\left(\vec{x}_{0}, t_{0}, \vec{\xi}_{1}\right) \\
\vdots \\
\alpha^{*}\left(\vec{x}_{0}, t_{0}, \vec{\xi}_{N P}\right)
\end{array}\right)
$$

The system can be solved by the least squares method. Once the coefficient $\alpha_{j}\left(\vec{x}_{0}, t_{0}\right)(j=0,1, \cdots, P)$ is obtained, statistics (means and standard deviations) can be estimated as shown in Eqs. (5) and (6).

The mean is given by

$$
\begin{aligned}
E\left[\alpha^{*}\left(\vec{x}_{0}, t_{0}, \vec{\xi}\right)\right] & =\int_{R} \alpha^{*}\left(\vec{x}_{0}, t_{0}, \vec{\xi}\right) w(\vec{\xi}) \mathrm{d} \vec{\xi} \\
& =\alpha_{0}\left(\vec{x}_{0}, t_{0}\right)
\end{aligned}
$$

which corresponds to the zeroth mode of expansion.

The weight function,

$$
w(\vec{\xi})=\frac{1}{\sqrt{2 \pi}} e^{-\frac{1}{2} \vec{\xi}^{\mathrm{T}} \vec{\xi}}
$$

is used in this study because the Gaussian distribution is assumed. $R$ is the support region of the weight function.

The variance is given by

$$
\begin{aligned}
& \operatorname{Var}\left[\alpha^{*}\left(\vec{x}_{0}, t_{0}, \vec{\xi}\right)\right] \\
& \quad=\int_{R}\left(\alpha^{*}\left(\vec{x}_{0}, t_{0}, \vec{\xi}\right)-\alpha_{0}\left(\vec{x}_{0}, t_{0}\right)\right)^{2} w(\vec{\xi}) \mathrm{d} \vec{\xi} \\
& \quad=\sum_{j=1}^{P}\left[\alpha_{j}^{2}\left(\vec{x}_{0}, t_{0}\right)\left\langle\Psi_{j}^{2}\right\rangle\right] .
\end{aligned}
$$

Here, the inner product of $\Psi_{i}(\vec{\xi})$ and $\Psi_{j}(\vec{\xi})$ in the support region is defined as follows:

$$
\begin{aligned}
\left\langle\Psi_{i}(\vec{\xi}) \Psi_{j}(\vec{\xi})\right\rangle & =\int_{R} \Psi_{i}(\vec{\xi}) \Psi_{j}(\vec{\xi}) w(\vec{\xi}) \mathrm{d} \vec{\xi} \\
& =\left\{\begin{array}{lll}
0 & \text { if } & i \neq j \\
\left\langle\Psi_{j}^{2}(\vec{\xi})\right\rangle & \text { if } & i=j .
\end{array}\right.
\end{aligned}
$$




\subsection{Validation}

The output behavior of the one-dimensional Burgers' equation with uncertainty in the boundary condition is estimated using NIPC to check the validity of the present method. The one-dimensional Burgers' equation used in this study is as follows

$$
\begin{aligned}
u_{t}+u u_{x} & =v u_{x x}, \quad x \in[-1,1] \\
u(x, 0) & = \begin{cases}1 & x \leq 0 \\
-1 & x \geq 0\end{cases} \\
u(-1, t) & =1+\delta, \quad u(1, t)=-1
\end{aligned}
$$

where $\delta$ is uncertainty in the boundary condition, which has a normal distribution and mean $(\mu)$ and standard deviation $(\sigma)$ are 0.05 and 0.025 , respectively. Thus, the actual value of $\delta$ used is $\mu \pm \xi \sigma$.

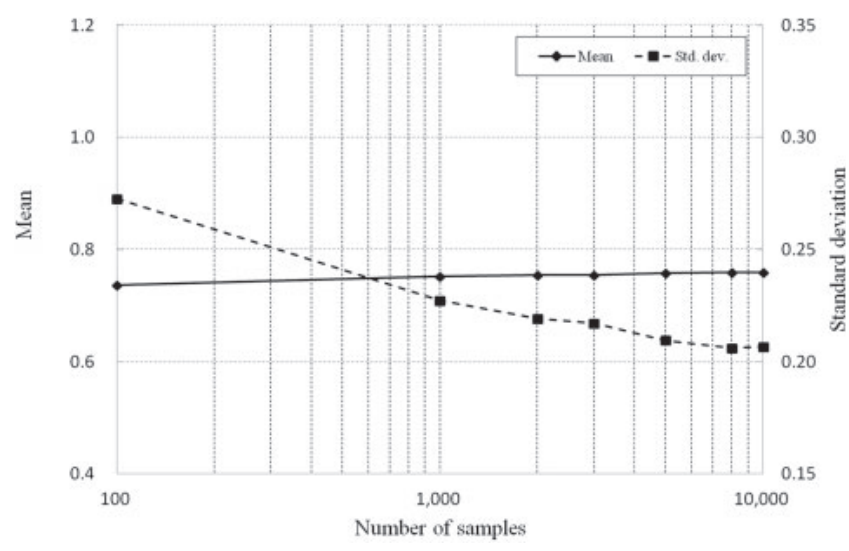

(a) Mean and standard deviation of Monte Carlo (MC)

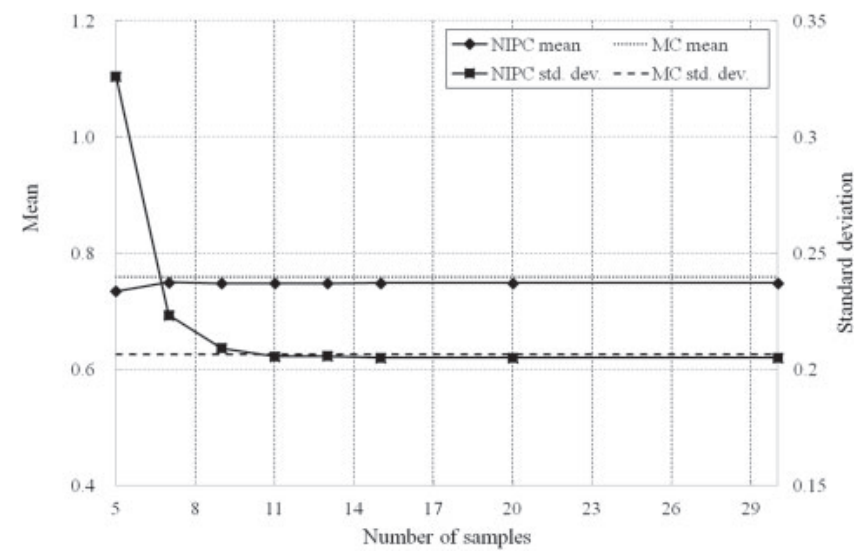

(b) Mean and standard deviation of non-intrusive polynomial chaos (NIPC)

Fig. 1. Convergence of mean and standard deviation.
The statistics of $\left.x\right|_{u=0}$ are estimated under conditions of uncertainty $\delta$ in the input boundary condition. Figure $1 \mathrm{com}-$ pares the mean and standard variation obtained by the Monte Carlo (MC) method and NIPC. The statistics of MC converged with approximately 10,000 samples, while those of NIPC converged with only 11 sample points. The values of mean and standard deviation of NIPC were well matched with those of MC. NIPC estimated the statistics accurately with $1 / 1,000$ the computational cost of MC.

Figure 2 shows the mean and the standard deviation of NIPC by varying the order of NIPC and range of sample points. In the range of $-5.0 \leq \xi \leq 5.0,11$ and 21 sample points are selected with the uniform interval, respectively. In the range of $-8.0 \leq \xi \leq 8.0,21$ sample points are selected with the uniform interval. With 11 sample points in the range of $-5.0 \leq \xi \leq 5.0$, the mean and the standard deviation of NIPC correspond to those of MC with a high order of polynomial. It seems that the order of polynomial of 7-10 is required for the range of $-5.0 \leq \xi \leq 5.0$. With 21 sample points in the range of $-5.0 \leq \xi \leq 5.0$, the mean and standard deviation of NIPC converged to those of MC with the order of polynomial of 9-14. With the order polynomial higher than 14, the standard deviation diverged. With 21 sample points in the range of $-8.0 \leq \xi \leq 8.0$, the mean and standard deviation of NIPC converged to those of MC with the order of polynomial of 16-20. Thus, in order to construct the accurate NIPC, the order of polynomial should be determined carefully according to the range of sample point distribution. In Table 2, $\xi$ and coefficients of NIPC $\left(\alpha_{j}, j=0,10\right)$ with 11 sample points are shown.

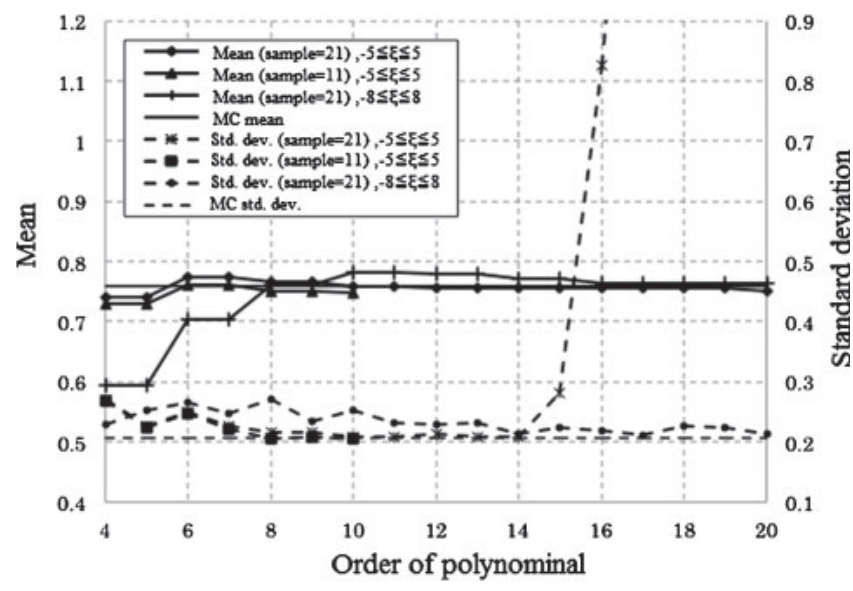

Fig. 2. Mean and standard deviation of NIPC according to order of polynomial.

Table 2. $\quad \xi$ and coefficients of NIPC $\left(\alpha_{j}, j=0,10\right)$ when $N P=11$.

\begin{tabular}{c|cccccc}
\hline$\xi$ & \multicolumn{5}{|c}{$-5.0,-4.0,-3.0,-2.0,-1.0,0,1.0,2.0,3.0,4.0,5.0$} \\
\hline \multirow{4}{*}{$\alpha_{j}$} & $j=0$ & $j=1$ & $j=2$ & $j=3$ & $j=4$ & $j=5$ \\
\cline { 2 - 7 } & 0.750322 & 0.129319 & $-8.85 \mathrm{E}-02$ & $3.28 \mathrm{E}-02$ & $-8.02 \mathrm{E}-04$ & $-4.01 \mathrm{E}-03$ \\
\cline { 2 - 7 } & $j=6$ & $j=7$ & $j=8$ & $j=9$ & $j=10$ & \\
\cline { 2 - 7 } & $1.04 \mathrm{E}-03$ & $2.25 \mathrm{E}-04$ & $-1.01 \mathrm{E}-04$ & $-7.22 \mathrm{E}-06$ & $8.88 \mathrm{E}-06$ & \\
\hline
\end{tabular}




\section{Sonic Boom Propagation Analysis}

\subsection{Augmented Burgers' equation}

The augmented Burgers' equation used to estimate sonic boom propagation in this study was as follows:

$$
\begin{aligned}
\frac{\partial p}{\partial x}= & \frac{\beta}{2 \rho_{0} c_{0}^{3}} \frac{\partial p^{2}}{\partial t^{\prime}}-\frac{1}{2 A} \frac{\partial A}{\partial x} p+\frac{1}{2 \rho_{0} c_{0}} \frac{\partial\left(\rho_{0} c_{0}\right)}{\partial x} p+\frac{\delta}{2 c_{0}^{3}} \frac{\partial^{2} p}{\partial t^{\prime 2}} \\
& +\sum_{i} \frac{(\Delta c)_{i} \tau_{i}}{c_{0}^{2}}\left(1+\tau_{i} \frac{\partial}{\partial t^{\prime}}\right)^{-1} \frac{\partial^{2} p}{\partial t^{\prime 2}}
\end{aligned}
$$

where $p=p\left(x, t^{\prime}\right)$ is the sound pressure, $x$ is the ray path distance, $A$ is the ray tube area, $c_{0}$ is the small-signal sound velocity, $\rho_{0}$ is the ambient density, $\beta$ is the coefficient of nonlinearity,

$$
t^{\prime}=t-\int_{0}^{x}\left(1 / c_{0}\right) \mathrm{d} x
$$

is the delayed time and $\delta$ is the diffusivity of sound due to both viscosity and heat conduction. Each relaxation process in atmosphere layer $i$ is characterized by a relaxation time $\tau_{i}$ and small-single sound speed increment $(\Delta c)_{i}$.

Equation (12) is solved using the finite difference method with the operator splitting method. ${ }^{11)}$ In this method, each term in Eq. (12) is solved one by one as follows

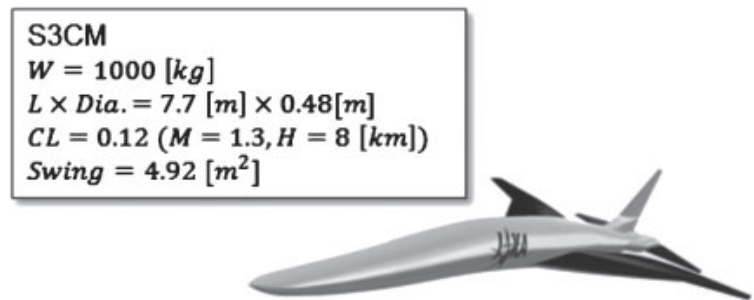

(a) D-SEND\#2 model

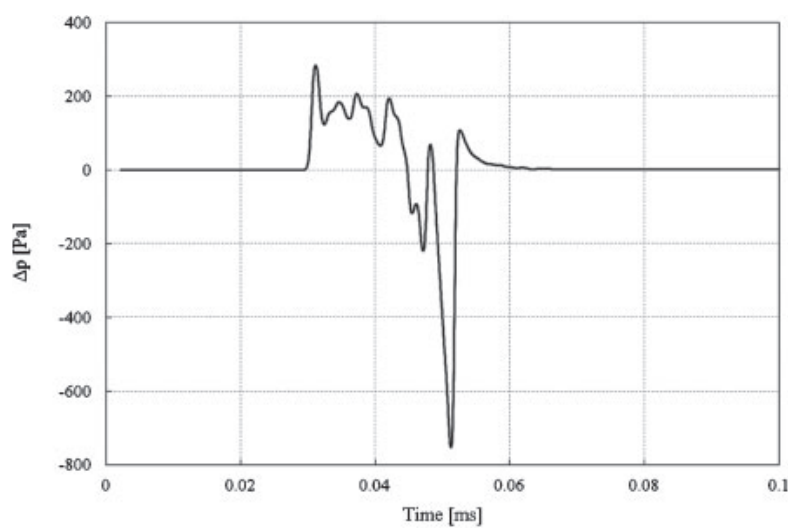

(b) Near-field pressure wave of D-SEND\#2 model

\begin{tabular}{|c|c|c|c|c|c|c|c|}
\hline \multirow{2}{*}{ Level } & \multirow{2}{*}{$\begin{array}{c}\text { Pressure } \\
(\mathrm{hPa})\end{array}$} & \multicolumn{2}{|c|}{ Geo-potential hgt. (m) } & \multicolumn{4}{|c|}{ Temperature (K) } \\
\hline & & Mean & Std. dev. & Mean & Std. dev. & Max. & Min. \\
\hline 1 & 1,000 & 90 & 65 & 286.4 & 3.5 & 297.8 & 275.8 \\
\hline 2 & 925 & 743 & 67 & 282.7 & 3.6 & 293.1 & 271.6 \\
\hline 3 & 850 & 1,440 & 70 & 278.4 & 3.6 & 288.8 & 267.3 \\
\hline 4 & 700 & 3,001 & 81 & 269.7 & 3.3 & 278.5 & 258.2 \\
\hline 5 & 600 & 4,205 & 93 & 262.8 & 3.5 & 271.9 & 249.3 \\
\hline 6 & 500 & 5,585 & 108 & 254.0 & 3.7 & 262.2 & 239.2 \\
\hline 7 & 400 & 7,210 & 130 & 242.3 & 3.9 & 251.6 & 228.6 \\
\hline 8 & 300 & 9,185 & 156 & 227.5 & 3.0 & 236.5 & 217.3 \\
\hline 9 & 250 & 10,384 & 160 & 222.0 & 3.0 & 232.6 & 214.8 \\
\hline 10 & 200 & 11,840 & 141 & 223.8 & 5.3 & 236.2 & 210.5 \\
\hline 11 & 150 & 13,739 & 116 & 225.3 & 3.2 & 232.7 & 214.8 \\
\hline 12 & 100 & 16,405 & 97 & 224.8 & 2.3 & 231.5 & 218.3 \\
\hline 13 & 70 & 18,753 & 88 & 224.7 & 1.8 & 229.9 & 219.2 \\
\hline 14 & 50 & 20,967 & 88 & 224.6 & 1.7 & 229.1 & 220.3 \\
\hline 15 & 30 & 24,330 & 100 & 225.5 & 2.0 & 230.4 & 220.8 \\
\hline 16 & 20 & 27,023 & 118 & 228.3 & 2.2 & 233.4 & 222.7 \\
\hline 17 & 10 & 31,737 & 161 & 235.6 & 2.8 & 242.8 & 227.6 \\
\hline
\end{tabular}

Fig. 3. D-SEND\#2 model and its near-field pressure wave.

Table 3. Temperature uncertainty in the atmosphere.

Table 4. Humidity uncertainty in the atmosphere.

\begin{tabular}{ccccccccc}
\hline \multirow{2}{*}{ Level } & \multirow{2}{*}{$\begin{array}{c}\text { Pressure } \\
(\mathrm{hPa})\end{array}$} & \multicolumn{2}{c}{ Geo-potential hgt. $(\mathrm{m})$} & & \multicolumn{3}{c}{ Specific humidity $(\mathrm{g} / \mathrm{kg})$} \\
\cline { 3 - 4 } \cline { 7 - 9 } & & Average & Std. dev. & & Average & Std. dev. & Max. & Min. \\
\hline 1 & 1,000 & 90 & 65 & & 7.938 & 1.828 & 13.970 & 3.751 \\
2 & 925 & 743 & 67 & & 6.414 & 1.582 & 12.059 & 2.452 \\
3 & 850 & 1,440 & 70 & & 4.934 & 1.292 & 9.848 & 0.887 \\
4 & 700 & 3,001 & 81 & & 2.672 & 1.077 & 6.474 & 0.123 \\
5 & 600 & 4,205 & 93 & & 1.592 & 0.812 & 4.452 & 0.000 \\
6 & 500 & 5,585 & 108 & & 0.848 & 0.491 & 2.943 & 0.000 \\
7 & 400 & 7,210 & 130 & & 0.360 & 0.243 & 1.668 & 0.000 \\
8 & 300 & 9,185 & 156 & & 0.103 & 0.078 & 0.502 & 0.000 \\
\hline
\end{tabular}


Table 5. Wind velocity uncertainty in the atmosphere.

\begin{tabular}{rrrrrrrrrr}
\hline \multirow{2}{*}{ Level } & \multirow{2}{*}{$\begin{array}{c}\text { Pressure } \\
(\mathrm{hPa})\end{array}$} & \multicolumn{2}{c}{ Geo-potential hgt. $(\mathrm{m})$} & & \multicolumn{4}{c}{ u-wind $(\mathrm{m} / \mathrm{s})$} \\
\cline { 3 - 4 } \cline { 7 - 9 } & Average & Std. dev. & & Average & Std. dev. & Max. & Min. \\
\hline 1 & 1,000 & 90 & 65 & & -0.11 & 3.27 & 13.15 & -9.80 \\
2 & 925 & 743 & 67 & & 0.92 & 4.29 & 16.02 & -13.20 \\
3 & 850 & 1,440 & 70 & & 2.27 & 4.50 & 16.89 & -11.89 \\
4 & 700 & 3,001 & 81 & & 3.74 & 5.12 & 21.99 & -11.34 \\
5 & 600 & 4,205 & 93 & & 4.75 & 5.88 & 25.18 & -12.71 \\
6 & 500 & 5,585 & 108 & & 6.06 & 7.01 & 28.51 & -13.75 \\
7 & 400 & 7,210 & 130 & & 7.74 & 8.71 & 35.99 & -18.77 \\
8 & 300 & 9,185 & 156 & & 9.61 & 11.07 & 48.65 & -25.27 \\
9 & 250 & 10,384 & 160 & & 9.97 & 11.34 & 50.43 & -25.13 \\
10 & 200 & 11,840 & 141 & & 8.50 & 8.88 & 41.78 & -15.62 \\
11 & 150 & 13,739 & 116 & & 6.45 & 5.58 & 25.44 & -9.28 \\
12 & 100 & 16,405 & 97 & & 4.22 & 3.61 & 17.01 & -5.91 \\
13 & 70 & 18,753 & 88 & & 2.40 & 2.81 & 11.76 & -5.57 \\
14 & 50 & 20,967 & 88 & & 0.72 & 2.51 & 8.61 & -5.58 \\
15 & 30 & 24,330 & 100 & & -1.60 & 2.38 & 5.83 & -8.37 \\
16 & 20 & 27,023 & 118 & & -2.67 & 2.40 & 4.85 & -9.95 \\
17 & 10 & 31,737 & 161 & & -3.43 & 3.37 & 9.64 & -13.61 \\
\hline
\end{tabular}

$\frac{\partial p}{\partial x}=\frac{\beta}{2 \rho_{0} c_{0}^{3}} \frac{\partial p^{2}}{\partial t^{\prime}}$

$\frac{\partial p}{\partial x}=-\frac{1}{2 A} \frac{\partial A}{\partial x} p$

$\frac{\partial p}{\partial x}=\frac{1}{2 \rho_{0} c_{0}} \frac{\partial\left(\rho_{0} c_{0}\right)}{\partial x} p$

$\frac{\partial p}{\partial x}=\frac{\delta}{2 c_{0}^{3}} \frac{\partial^{2} p}{\partial t^{\prime 2}}$,

$$
\frac{\partial p}{\partial x}=\frac{(\Delta c)_{i} \tau_{i}}{c_{0}^{2}}\left(1+\tau_{i} \frac{\partial}{\partial t^{\prime}}\right)^{-1} \frac{\partial^{2} p}{\partial t^{\prime 2}}, \quad i=1,2, \cdots
$$

or

$$
\left(1+\tau_{i} \frac{\partial}{\partial t^{\prime}}\right) \frac{\partial p}{\partial x}=\frac{(\Delta c)_{i} \tau_{i}}{c_{0}^{2}} \frac{\partial^{2} p}{\partial t^{\prime 2}}, \quad i=1,2, \cdots
$$

Equations (13)-(17) are solved separately at each matching step $\Delta x$. For the nonlinear term in Eq. (13), the upwind difference is employed and the ray tube area $A$ in Eq. (14) is evaluated using the ray tracing method. For the thermoviscous and relaxation term in Eqs. (16) and (17), the second-order time derivatives are evaluated using central difference. The Crank-Nicolson method is used for space integration.

Equations (13)-(15) are related with the nonlinear effect, geometric damping effect and stratification effect (atmospheric conditions change according to altitude), respectively. Equations (16) and (17) take into account the thermal viscosity attenuation and relaxation attenuation, respectively. These two terms make it possible to estimate the sonic boom rise time.

\subsection{Near-field pressure distribution and atmospheric uncertainty}

The near-field pressure wave used in this study is that of the D-SEND\#2 model, which was developed in the JAXA

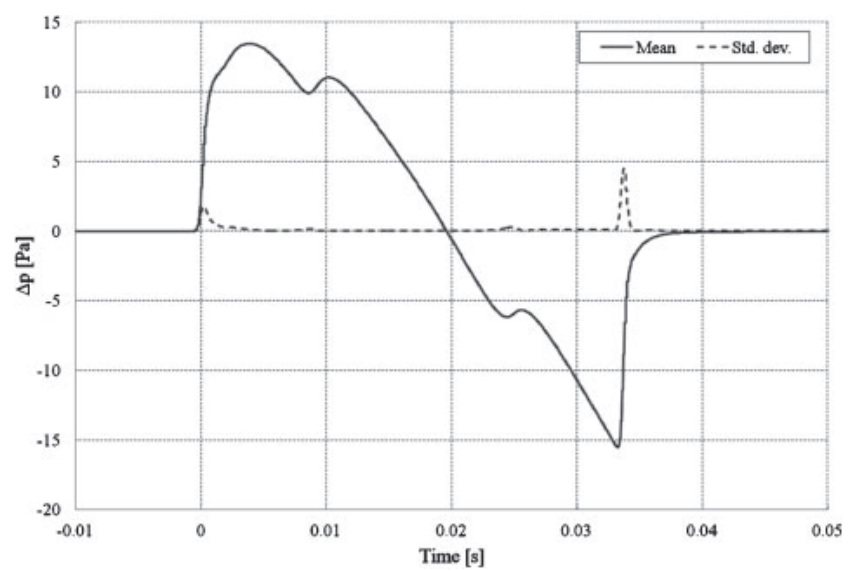

(a) Monte Carlo

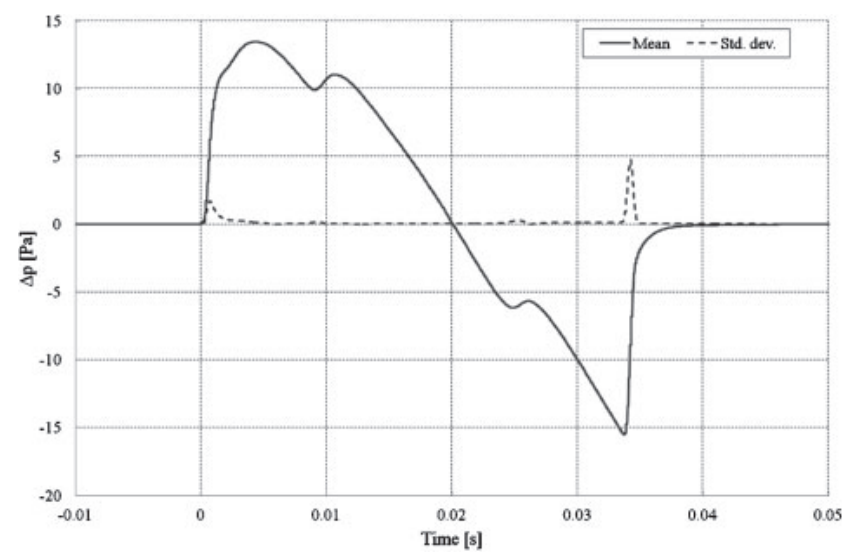

(b) NIPC

Fig. 4. Mean and standard deviation of sonic boom pressure. 


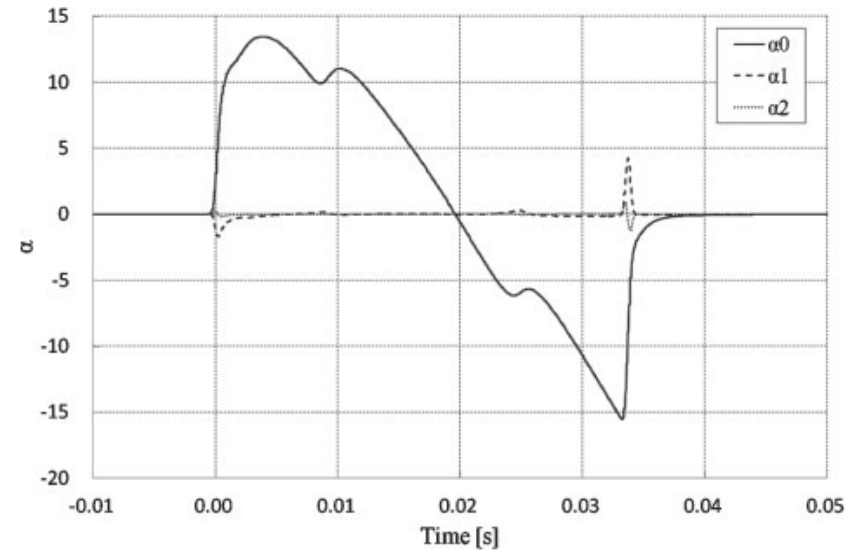

(a) Coefficients of NIPC $\left(\alpha_{0}-\alpha_{2}\right)$

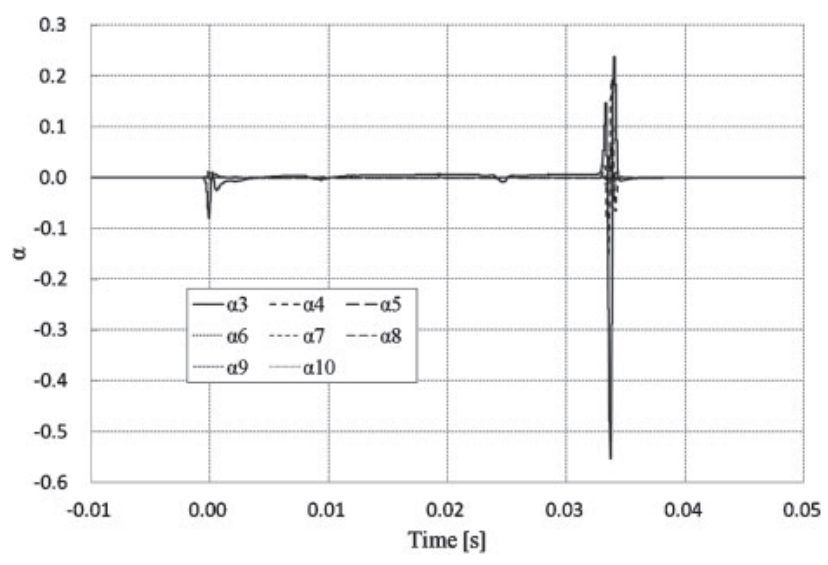

(b) Coefficient of NIPC $\left(\alpha_{3}-\alpha_{10}\right)$

Fig. 5. Coefficients of NIPC with 11 sample points.

D-SEND (drop test for simplified evaluation of non-symmetrically distributed sonic boom) project as shown in Fig. 3. This near-field pressure wave is measured at a location twice the length of the model below the model axis. The flight altitude, Mach number and flight path angle of D-SEND\#2 are 8,000 [m], 1.3 and -50 [deg], respectively.

The present analysis considers the temperature, wind (speed and direction) and humidity as factors of uncertainty in the atmosphere, and the database of measurements in August from 2000 to 2009 at the Esrange Space Center are used.

For the uncertainty of atmospheric temperature and wind velocity, the altitudes from 98 to 31,376 [m] are divided into 17 layers. For the uncertainty of atmospheric humidity, the altitudes from 90 to 9,185 [m] are divided into eight layers. The mean $(\mu)$ and standard deviation $(\sigma)$ of atmospheric properties are given in each layer separately as shown in Tables 3-5. The geo-potential height in the tables is the height corresponding to specific pressure $(10-1,000 \mathrm{hPa})$. Thus, the layers are actually divided at the height where its pressure coincides with a specific value. The values of atmospheric temperature, humidity and wind velocity in the tables are also the values at the specific pressure. The uncertainty of these properties is represented as $\mu+\xi \sigma$, where $\xi$ is a normally distributed random variable with a

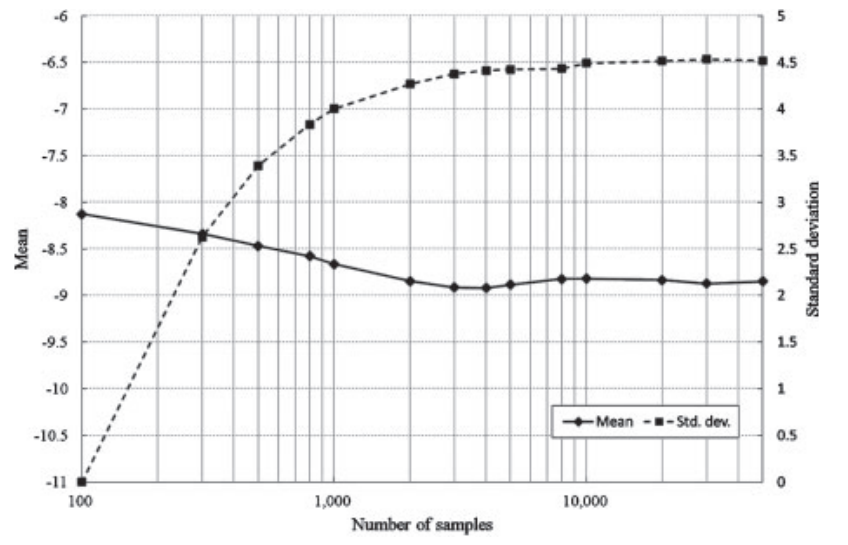

(a) Mean and standard deviation of Monte Carlo method

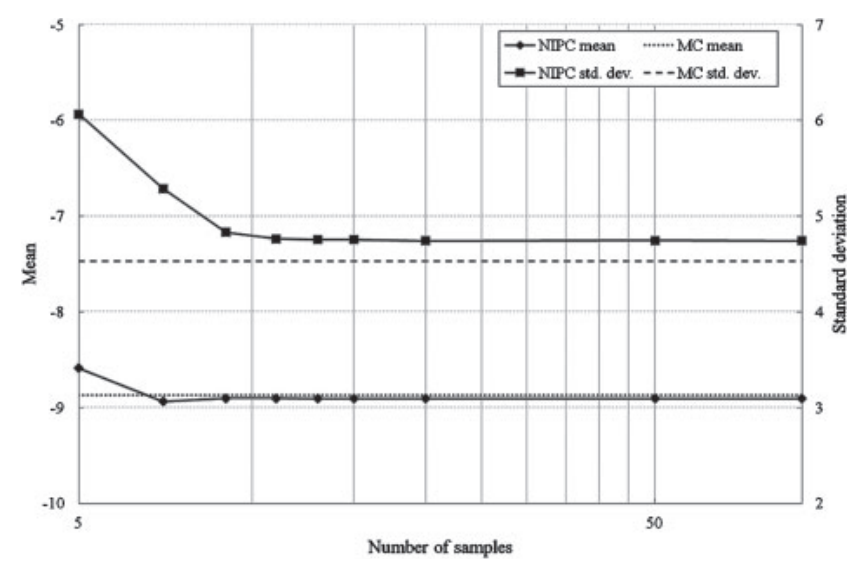

(b) Mean and standard deviation of NIPC

Fig. 6. Convergence of mean and standard deviation of sonic boom pressure at $t=0.034$.

mean value and standard deviation of 0 and 1 , respectively. Once the value of $\xi$ is determined for one sample, the same value of $\xi$ is applied to all layers. If $\mu+\xi \sigma$ of humidity in the layer is negative, the humidity is set to zero.

\section{Results}

\subsection{Validation of NIPC for sonic boom propagation}

To confirm the NIPC for sonic boom propagation under conditions of atmospheric uncertainty, the statistical behavior of sonic boom propagation under conditions of atmospheric temperature uncertainty are estimated using both NIPC and MC. Figure 4 shows the means and standard deviations of sonic boom pressure along the time obtained using NIPC and MC. The number of sample points for NIPC and MC are 11 and 10,000, respectively. The means of sonic boom pressure obtained using both methods matched very well. The times at the two peaks of standard deviation ( $t=0.0$ and $t=0.034)$ also corresponded to each other. The coefficients of NIPC $\left(\alpha_{j}, j=0,10\right)$ with 11 sample points $(\xi= \pm 5, \pm 4, \pm 3$, $\pm 2, \pm 1,0)$ are shown in Fig. 5 ( $\alpha_{0}$ corresponds to the mean in Fig. 4(b)).

Figure 6 shows the convergence of statistics at $t=0.034$ when the standard deviation of sonic boom pressure is the 


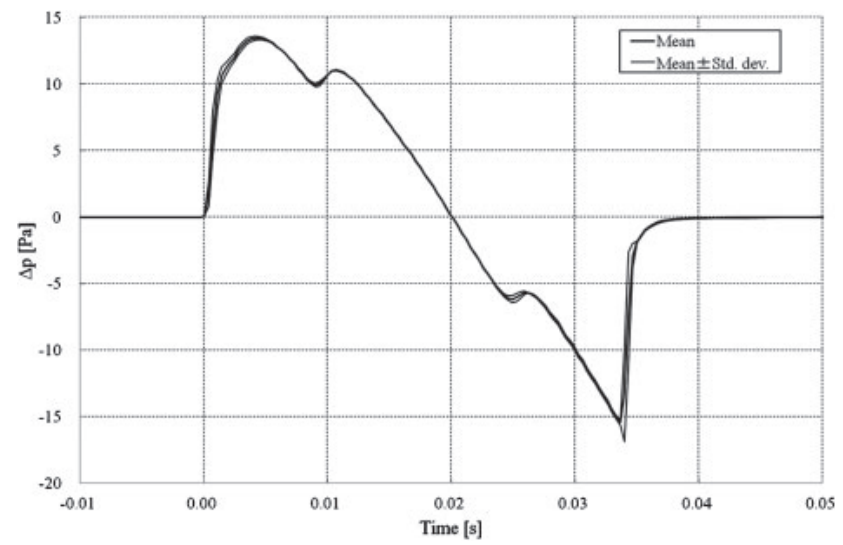

Fig. 7. Sonic boom signature under conditions of temperature uncertainty.

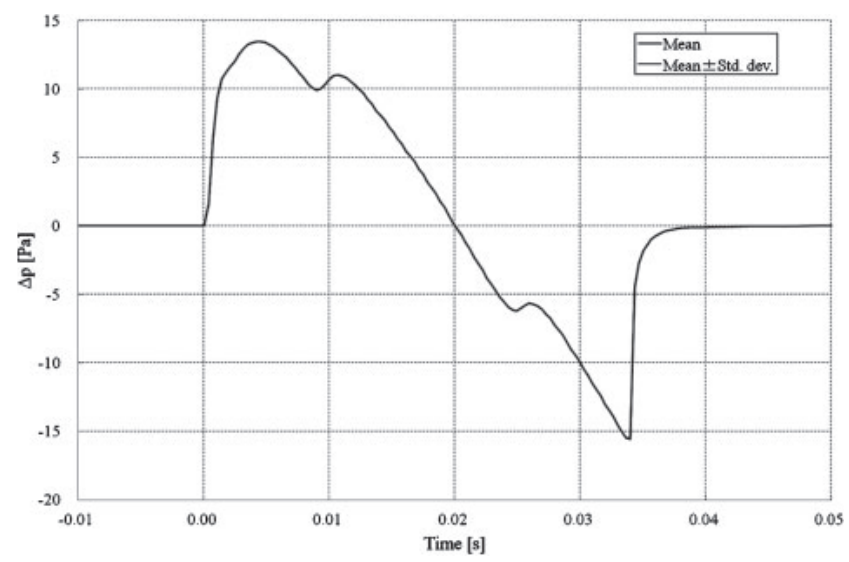

Fig. 8. Sonic boom signature under conditions of humidity uncertainty.

greatest. The statistics of MC converge with approximately 10,000 samples, while those of NIPC converge with 11 samples. The difference in statistics between MC and NIPC is less than 5\%. Thus, it was confirmed that the NIPC has sufficient accuracy for estimation of the statistical behavior of sonic boom propagation.

\subsection{Sonic boom signature under conditions of atmo- spheric temperature, humidity and wind velocity uncertainty}

Figure 7 shows the means and the standard deviations of sonic boom signature under conditions of uncertainty in atmospheric temperature obtained using NIPC with 11 samples. The thick line indicates the mean of sonic boom pressure and thin lines indicate mean \pm std. dev, respectively. On the whole, the standard deviations at which the positive and negative pressure peaks are located are relatively large. This is because of the effect of thermal viscosity under conditions of uncertainty in atmospheric temperature.

Figure 8 shows the mean and standard deviation of sonic boom signature under conditions of uncertainty in atmospheric humidity. Compared with those of temperature, the standard deviation is relatively large throughout the entire sonic boom signature. This large standard deviation is attributable to the relaxation effect of humidity. Figure 9 shows the means and standard deviations of sonic boom signature under conditions of uncertainty in wind velocity. The

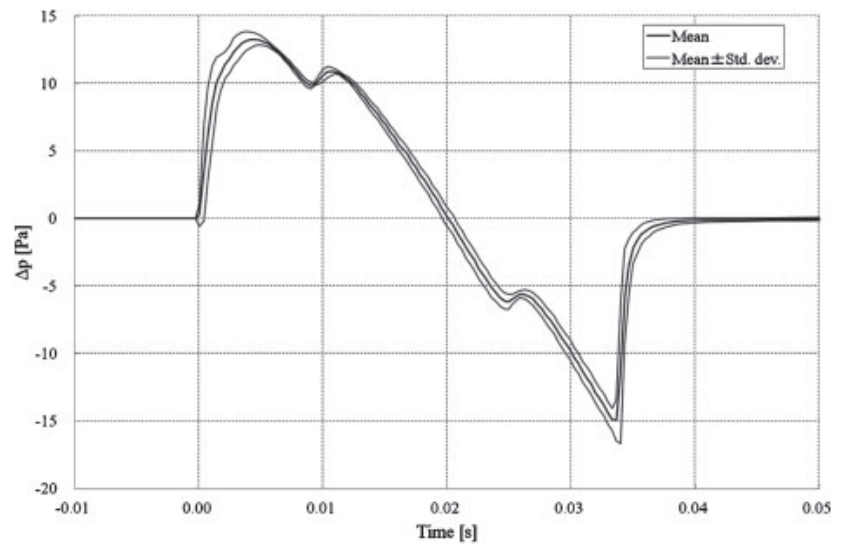

Fig. 9. Sonic boom signature under conditions of wind velocity uncertainty.

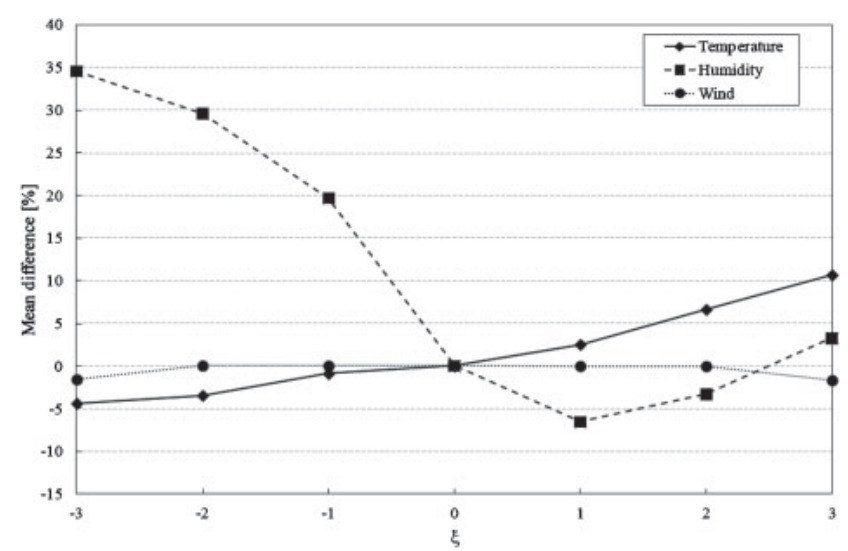

(a) Rise time

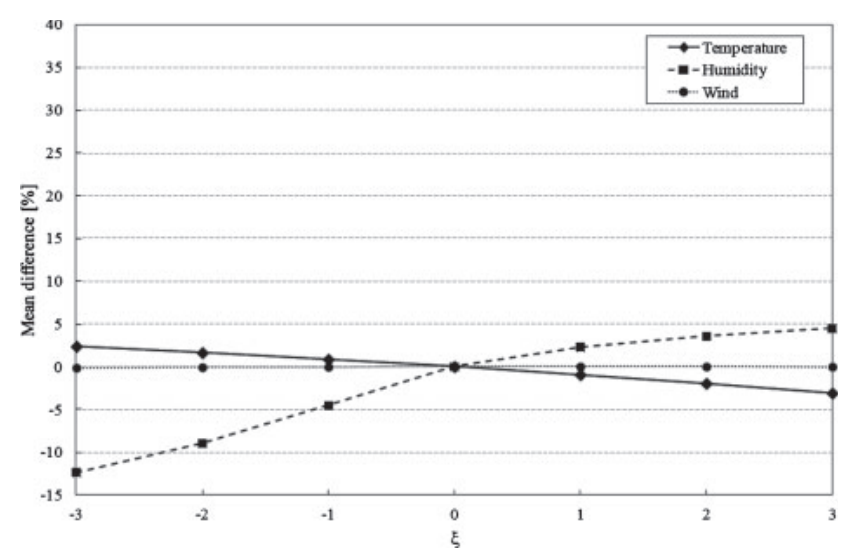

(b) Peak pressure

Fig. 10. Difference of mean value according to disturbance.

standard deviation is very small throughout the entire boom signature. The reason for this small standard deviation is that the same value of disturbance $(\xi)$ was applied to all layers in this study, which makes it difficult to account for the effects of turbulence in atmosphere layers.

Figure 10 shows differences in peak pressure and rise time from the means according to the amount of disturbance $(\xi)$. The rise time is affected by both temperature and humidity, while peak pressure is affected only by humidity. 


\section{Conclusions}

In this study, NIPC, which is able to estimate statistics under conditions of uncertainty at low computational cost, was adopted for sonic boom analysis using an augmented Burgers' equation. The present method predicted the statistics of sonic boom strength and rise time with equivalent accuracy and much smaller sample size compared to the Monte Carlo (MC) method. The results showed that temperature uncertainty has an impact on the local rise in sonic boom pressure, and atmospheric humidity uncertainty has an impact on the entire shape of the sonic boom signature, while wind uncertainty has almost no impact on sonic boom propagation.

\section{Acknowledgments}

The authors would like to thank the supersonic transport team at JAXA for providing the atmospheric data in the D-SEND project and Mr. Masafumi Yamamoto of RCCM for providing technical support in the Burgers' code.

\section{References}

1) Thomas, C. L.: Extrapolation of Sonic Boom Pressure Signatures by the Waveform Parameter Methods, NASA TN D-6832, 1972.
2) Cleveland, R. O. and Blackstock, D. T.: Waveform Freezing of Sonic Booms Revisited, NASA CP-3335, 1996.

3) Pierce, A. D.: Acoustics-An Introduction to Its Physical Principles and Application, Acoustical Society of America, New York, 1991.

4) George, S. F.: Monte Carlo: Concepts, Algorithms, and Applications, Springer-Verlag, New York, 1996.

5) Hosder, S. and Walters, R. W.: Non-intrusive Polynomial Chaos Uncertainty Quantification in Fluid Dynamics, AIAA Aeropace Sciences Meeting Including the New Horizons Forum and Aerospace Exhibition, Orando, Florida, 2010.

6) Xiu, D. and Karniadakis, G. E.: Modeling Uncertainties in Flow Simulation via Generalized Polynomial Chaos, J. Comput. Phys., 187 (2003), pp. 137-167.

7) Khodaparast, H. H., Mottershead, J. E. and Friswell, M. I.: Perturbation Methods for the Estimation of Parameter Variability in Stochastic Model Updating, Mech. Syst. Signal Proc., 22 (2008), pp. 1751-1773.

8) Hua, X. G., Ni, Y. Q., Chen, Z. Q. and Jo, J. M.: An Improved Pertubation Method for Stochastic Finite Element Model Updating, Int. J. Numer. Methods Eng., 73 (2008), pp. 1845-1864.

9) Huyse, L.: Free-form Airfoil Shape Optimization under Uncertainty Using Maximum Expected Value and Second-order Second-moment Strategies, ICASE Report No. 2001-18, NASA CR 2001-211020, 2001.

10) Padulo, M., Maginot, J. and Guenov, M.: Airfoil Design under Uncertainty with Robust Geometric Parameterization, 50th AIAA/ASME/ ASCE/AHS/ASC Structures, Structural Dynamics and Material Conference, 2009

11) Yamamoto, M., Hashimoto, A., Takahashi, T., Kamamura, T. and Sakai, T.: Long-Range Sonic Boom Prediction Considering Atmospheric Effects, InterNoise 2011, Osaka, 2011. 